\title{
Five-year results of the prospective, randomized, multicenter, Food and Drug Administration investigational device exemption study of the ProDisc-L total disc replacement versus circumferential arthrodesis for the treatment of single-level degenerative disc disease
}

\author{
Clinical article
}

\author{
JACK E. Zigler, M.D., ${ }^{1}$ ANd Rick B. Delamarter, M.D. ${ }^{2}$ \\ ${ }^{1}$ Texas Back Institute, Plano, Texas; and ${ }^{2}$ The Spine Institute at Saint John's Health Center, Santa Monica, California
}

\begin{abstract}
Object. The purpose of this study was to evaluate the long-term safety and effectiveness of the ProDisc-L total disc replacement (TDR) as part of an FDA-mandated postmarket approval study. This report summarizes the clinical findings after 5 years of follow-up.

Methods. Two hundred thirty-six patients were treated and followed up for 5 years; 161 TDRs and 75 fusions had been performed in these patients. The primary outcome was a 10-component success end point. Secondary outcome measures included neurological status, secondary surgery, Oswestry Disability Index (ODI), 36-Item Short Form Health Survey (SF-36), visual analog scale (VAS) assessing pain and satisfaction, radiographic data, narcotic use, activity, and recreation status. Patients were monitored through their 5-year postoperative visits under the FDA postmarket surveillance provisions in the original investigational device exemption approval.

Results. The overall follow-up rate at 5 years was $81.8 \%$. Study success demonstrated that TDR was noninferior to fusion with a $12.5 \%$ margin $(\mathrm{p}=0.0099)$. Both TDR and fusion treatment groups maintained significant improvement on the ODI at 5 years compared with baseline $(\mathrm{p}<0.0001)$. Secondary surgeries at the index level were performed in $12 \%$ of fusion patients and $8 \%$ of TDR patients. Radiographically, none of the TDRs developed spontaneous fusion. The segmental range of motion following TDR remained within normal range, although it decreased by approximately $0.5^{\circ}$ in years 3 to 5 . The VAS pain scores decreased from preoperative values by $48 \%$ in both treatment groups at 5 years. Patient satisfaction remained high in both groups (77\%), while the percentage of patients indicating that they would have the surgery again was higher in TDR patients $(82.5 \%)$ than in fusion patients $(68.0 \%)$.

Conclusions. Patients in both groups maintained significant improvement during the 5-year follow-up. The TDR group had significantly better improvement on some scales. Although TDR patients avoid the stiffness of fusion and are more satisfied than fusion patients, both fusion and TDR are reasonable surgical options in this specific patient population. (http://thejns.org/doi/abs/10.3171/2012.9.SPINE11498)
\end{abstract}

\section{Key Words - total disc replacement • lumbar spine • degenerative • randomized study $\quad$ clinical outcome}

$\mathrm{S}$ URGICAL treatment of patients with mechanical DDD has been controversial, but improvements in clinical outcomes have been shown in properly selected patients,${ }^{6,8,9}$ with fusion arguably becoming the gold-standard choice for managing these patients. One potential drawback is the fact that a fused lumbar segment alters the biomechanics of the remaining, often healthy, spinal segments. Total disc replacement technologies have been

\footnotetext{
Abbreviations used in this paper: DDD = degenerative disc disease; IDE = investigational device exemption; ODI = Oswestry Disability Index; ROM = range of motion; SF-36 PCS = 36-Item Short Form Health Survey physical component score; TDR = total disc replacement; VAS = visual analog scale.
}

developed as an alternative to preserve motion at the affected level for functionally disabling discogenic back pain. The ProDisc-L (Synthes Spine, Inc.) is one such TDR device that has FDA approval in the US based on 2-year outcomes.

The purpose of this study was to evaluate the longterm safety and effectiveness of the ProDisc-L TDR. We previously reported the results of a 2-year IDE study. ${ }^{11}$ Continued follow-up of the patients in that study was conducted as part of an FDA-mandated postmarket approval study; this report summarizes the clinical findings after a minimum of 5 years of follow-up. The primary hypothesis of the present study was that TDR would be statistically noninferior to fusion. 


\section{Study Background}

\section{Methods}

Seventeen sites participated in the study. The first 3 patients enrolled at each site were nonrandomized training cases and were treated with TDR (51 enrolled, 50 treated). These patients were monitored in exactly the same clinical and radiographic manner as the randomized patients. Subsequent patients at each site were randomized in a 2:1 ratio of TDR to circumferential fusion. Patients were blinded to randomization until immediately postsurgery. Two hundred forty-two patients were randomized and treated. Six patients were treated off protocol based on intraoperative decisions made by their surgeons. Two of the cases involved unrecognized spondylotic defects not recognized preoperatively. One patient randomized to TDR was treated with TDR despite the index-level pars defect. The second patient was randomized to fusion, and the surgeon was not comfortable fusing adjacent to the newly recognized open-pars segment, so he performed a 2-level fusion. Four cases were administrative errors in that patients with 2-level involvement were scheduled for 2-level surgery but never disenrolled when the second level was indicated preoperatively. As these 6 patients were all enrolled with known exclusions, they were classified as "treated off protocol" and their data were not added to the study database.

An additional exclusion criterion relating to patient vertebral endplate size was added after the study began, so that in small-framed patients, investigators were asked to evaluate axial CT scans and exclude patients whose vertebral endplate dimensions were less than $34.5 \mathrm{~mm}$ in the mediolateral direction and/or less than $27 \mathrm{~mm}$ in the anteroposterior direction (the exact size of the smallest available ProDisc-L endplate).

The surgical procedure has been described in detail elsewhere. ${ }^{11}$ The fusion group received commercially available femoral ring allograft and posterolateral fusion with autogenous iliac crest bone graft in combination with pedicle screws. Although iliac crest harvest might be responsible for persistent outcome differences between the groups, this was not observed. No adverse events related to graft harvest (hematoma or infection) were encountered. No structural bone grafts were needed, as only cancellous bone was required for posterolateral onlay fusion, limiting the scope of crest violation, and graft was generally harvested through the same skin incision as the fusion.

No adverse events specific to iliac crest harvest were recorded. Although iliac crest pain certainly occurred, it was not believed to exert a clinically significant effect on the fusion patient's postoperative course. Even if iliac crest site pain contributed to increased pain (VAS) or impairment (ODI), this additional burden would normally be expected to resolve within a few weeks or months. At the 2-year and certainly the 5-year follow-up, pain from the iliac crest donor site would be expected to be insignificant.

Patients in all cohorts were evaluated preoperatively and at 6 weeks and 3,6,12,18, and 24 months postoperatively. After completing 24 months of follow-up, patients were asked to consent to additional follow-up at 36, 48, and 60 months. At each follow-up visit, physical and neu- rological examinations, radiography studies, and patient self-assessments were conducted. The self-assessments included the ODI, ${ }^{7}$ the SF-36, pain intensity on a $100-\mathrm{mm}$ VAS (VAS pain), patient satisfaction (VAS satisfaction), and whether the patient would have the same surgery again. For the purposes of FDA evaluation, a composite binary end point was defined in the protocol. The composite measure, termed "statistical success," encompassed the primary effectiveness and safety criteria summarized in Table 1. A patient was classified as a statistical success if and only if all criteria specified in the table were met.

Radiographs (anteroposterior and lateral, flexion and extension) were obtained preoperatively and at each follow-up visit, with patients standing when clinically possible to ensure their natural anatomical position. An independent radiographic reviewer (Medical Metrics, Inc.) who was blinded to the particular patient history or outcome and had no contact with any investigator both measured and analyzed radiographic data.

\section{Statistical Analysis}

Before initiating the study, sample size for the 24-month follow-up was estimated using the original approximate calculation of Blackwelder. ${ }^{1}$ A Type I error of $5 \%$ (1-sided) and $80 \%$ power was chosen, assuming an $85 \%$ chance of statistical success at 24 months in each group. The calculated sample size was 144 and 72 for the TDR and fusion groups, respectively, and 255 total (170 TDR and 85 fusion control) with a correction for $15 \%$ attrition.

The noninferiority hypothesis was based on an exact 95\% 1-sided upper confidence bound for the difference in overall statistical success probabilities between TDR and fusion groups. If the upper bound was within the noninferiority margin $(12.5 \%)$, then TDR was considered noninferior to fusion. All patients with available information at the 24- and 60-month follow-up visits were included in the noninferiority analysis, with the exception of the 6 protocol violators (Table 2).

Clinical outcomes as measured by the ODI, the VAS pain, the SF-36, the VAS satisfaction, surgery again, and adverse event rates are reported using a per-protocol analysis. This analysis excluded patients at a given follow-up if the intended treatment was significantly altered-specifically, the 6 patients treated off protocol, TDR patients who underwent index-level revisions, and fusion patients who underwent index-level revisions (excluding hardware removal after successful fusion). The per-protocol analysis represents data requested by and reported to the FDA. Note, however, that all patients were included in an intentto-treat analysis and will be discussed below.

Demographic data were compared in the TDR versus fusion cohorts using the Wilcoxon rank-sum test or Fisher exact test. Secondary clinical outcomes were compared in TDR versus fusion patients using 2-sample t-tests (percent improvements in ODI, VAS pain, SF-36, and VAS satisfaction) or Fisher exact test (components of statistical success, narcotics use, and recreational status). Data at 2 and 5 years after treatment were compared with baseline data within each treatment group using the paired t-test. Significance was set at $\mathrm{p}<0.05$. 
Five-year results of an FDA investigational device exemption study

TABLE 1: Ten criteria for composite binary end point of statistical success for FDA evaluation*

\begin{tabular}{|c|c|c|}
\hline Parameter & Fusion & TDR \\
\hline ODI score & $\geq 15 \%$ improvement from baseline & $\geq 15 \%$ improvement from baseline \\
\hline $\begin{array}{l}\text { no secondary surgical intervention } \\
\text { at index level }\end{array}$ & $\begin{array}{l}\text { no reop to modify fusion site or correct complication w/ im- } \\
\text { plant }\end{array}$ & no reop required to remove or modify TDR implant \\
\hline SF-36 PCS & 60 -mo score - baseline score $\geq 0$ & 60 -mo score - baseline score $\geq 0$ \\
\hline neurological status & $\begin{array}{l}\text { improved or maintained motor, sensory, \& reflex status \& } \\
\text { straight-leg raise }\end{array}$ & $\begin{array}{l}\text { improved or maintained motor, sensory, \& reflex sta- } \\
\text { tus \& straight-leg raise }\end{array}$ \\
\hline \multicolumn{3}{|l|}{ radiographic success } \\
\hline device migration & $\leq 3 \mathrm{~mm}$ & $\leq 3 \mathrm{~mm}$ \\
\hline device subsidence & $\leq 3 \mathrm{~mm}$ & $\leq 3 \mathrm{~mm}$ \\
\hline radiolucency & no halos or radiolucencies around implant & $\begin{array}{l}\text { no radiolucency along implant-bone interface }>25 \% \\
\text { of interface's length for each endplate }\end{array}$ \\
\hline flexion/extension ROM & $<3 \mathrm{~mm}$ translation $\&<5^{\circ}$ angulation & $\geq 6^{\circ}$ at $\mathrm{L} 3-4$ or $\mathrm{L} 4-5, \geq 5^{\circ}$ at $\mathrm{L} 5-\mathrm{S} 1$ \\
\hline disc height & loss of disc height $\leq 3 \mathrm{~mm}$ & loss of disc height $\leq 3 \mathrm{~mm}$ \\
\hline fusion status & $\begin{array}{l}\text { strong evidence of fusion, including }>50 \% \text { trabecular } \\
\text { bridging bone or bone mass maturation \& increased or } \\
\text { maintained bone density at site w/ no visible gaps in } \\
\text { fusion mass }\end{array}$ & no evidence of bony fusion \\
\hline
\end{tabular}

\footnotetext{
* A patient was considered a statistical success if and only if all of the criteria were met. A modified version of this table was published in Semin Spine Surg, Vol 24, Zigler JE, Five-year results of the ProDisc-L multicenter, prospective, randomized, controlled trial comparing ProDisc-L with circumferential spinal fusion for single-level disabling degenerative disk disease, 25-31, Copyright Elsevier, 2012.
}

\section{Results}

Table 2 summarizes patient enrollment and followup. Follow-up rates are reported for all patients who were evaluated at that follow-up visit, regardless of whether their data set was complete. Overall patient follow-up was $97.0 \%$ of 236 patients at 2 years and $81.8 \%$ of 236 patients at 5 years. Complete radiographic data sets were available for $72.9 \%$ of the patients. Demographics for patients enrolled and treated in the IDE study are shown in Table 3. There were no statistically significant differences between the fusion and TDR patients in any of the demographic characteristics, including age, body mass index, race, smoking status, baseline ODI, or percentage of patients who had prior surgical treatment.

Intraoperative data were previously reported in detail. ${ }^{11}$ The TDR group underwent an anterior approach, whereas the fusion group underwent anterior and posterior approaches. The mean intraoperative time was significantly shorter in the TDR group than in the fusion group (121.7 vs 229.0 minutes, $\mathrm{p}<0.0001)$. The mean estimated blood loss was lower in the TDR group than in the fusion control group (203.7 vs $466.8 \mathrm{ml}, \mathrm{p}<0.0001)$. Duration of hospital stay was also statistically significantly shorter in the TDR group (3.5 vs 4.3 days, $\mathrm{p}<0.0001)$ than in the control group.

\section{Primary Clinical Outcomes}

Composite End Point. For FDA evaluation, a composite outcome was determined based on criteria in Table 1, in which patients had to satisfy all criteria outlined to be a statistical success. At 2 years posttreatment, $63.5 \%$ of 148 TDR patients and $45.1 \%$ of 71 fusion patients achieved overall statistical success, supporting the hypothesis that TDR was noninferior to fusion with a $12.5 \%$ margin $(\mathrm{p}<0.0099)$. Although the study was not designed to show a difference, a statistically significant difference in success rates between the TDR and fusion groups at 2 years was found using a 1-sided Fisher exact test $(\mathrm{p}=0.0053)$. At 5 years posttreatment, $53.7 \%$ of 134 TDR patients and $50.0 \%$ of 52 fusion patients were classified as statistical successes. The primary hypothesis of our study - that TDR was noninferior to fusion-was supported with these 5-year data using a $12.5 \%$ margin $(\mathrm{p}=0.0236)$. To evaluate impact on results, which included any missing data, an intent-to-treat analysis was performed and included protocol violators as well as last available visit data. Noninferiority was still demonstrated while including all of these patients (34 [42.5\%] of 80 patients for fusion and 84 [51.8\%] of 162 patients for TDR, $\mathrm{p}=0.0008$ ). Superiority of TDR over fusion using this composite outcome success model was not demonstrated at 5 years $(\mathrm{p}=0.7438)$.

Results regarding each component of the composite end point are detailed in the sections that follow. Patients in whom secondary surgeries had failed were excluded from determinations of ODI, SF-36, neurological, and radiographic success outcomes because they no longer had the original treatment.

Oswestry Disability Index. Preoperative ODI scores of $63 \%$ were similar between fusion and TDR patients (Table 4). At 2 years posttreatment, ODI scores improved significantly as compared with baseline in both fusion and TDR patients $(\mathrm{p}<0.0001)$. The mean ODI score percentage improvements were greater in TDR patients than in fusion patients at 2 years $(\mathrm{p}=0.0550)$. A significantly greater number of TDR patients had $\geq 15 \%$ improvement 
TABLE 2: Summary of patient treatment allocation and follow-up*

\begin{tabular}{|c|c|c|}
\hline Period & Fusion Group & TDR Group \\
\hline allocation & $\begin{array}{l}93 \text { enrolled } \\
75 \text { received intervention } \\
18 \text { did not receive intervention } \\
2 \text { denied insurance coverage } \\
8 \text { changed mind } \\
3 \text { no longer surgical candidates at time of surgery } \\
5 \text { treated off protocol }\end{array}$ & $\begin{array}{l}183 \text { enrolled } \\
161 \text { received intervention } \\
22 \text { did not receive intervention } \\
13 \text { denied insurance coverage } \\
9 \text { changed mind } \\
1 \text { treated off protocol }\end{array}$ \\
\hline 2-yr FU† & $\begin{array}{l}73 \text { evaluated ( } 97.1 \% \mathrm{FU}) \\
2 \text { secondary surgical interventions (included in primary com- } \\
\text { posite end point; excluded from secondary analyses) } \\
2 \text { missed visit (excluded from analysis) }\end{array}$ & $\begin{array}{l}156 \text { evaluated ( } 96.9 \% \mathrm{FU}) \\
6 \text { secondary surgical interventions (included in primary composite end } \\
\text { point; excluded from secondary analyses) } \\
5 \text { missed visit (excluded from analysis) }\end{array}$ \\
\hline 5-yr FU‡ & $\begin{array}{l}56 \text { evaluated }(74.7 \% \mathrm{FU}) \\
5 \text { secondary surgical interventions (included in primary com- } \\
\text { posite end point, excluded from secondary analyses) } \\
19 \text { missed visits (excluded from analysis) } \\
1 \text { death } \\
18 \text { lost to FU }\end{array}$ & $\begin{array}{l}137 \text { evaluated ( } 85.1 \% \text { FU) } \\
11 \text { secondary surgical interventions (included in primary composite end } \\
\text { point, excluded from secondary analyses) } \\
24 \text { missed visits (excluded from analysis) } \\
4 \text { deaths } \\
20 \text { lost to FU }\end{array}$ \\
\hline
\end{tabular}

* A modified version of this table was published in Semin Spine Surg, Vol 24, Zigler JE, Five-year results of the ProDisc-L multicenter, prospective, randomized, controlled trial comparing ProDisc-L with circumferential spinal fusion for single-level disabling degenerative disk disease, 25-31, Copyright Elsevier, 2012. Values expressed as means \pm standard deviations, unless indicated otherwise. Abbreviation: FU = follow-up.

$\dagger$ Overall follow-up at 2 years: $97.0 \%$.

$\ddagger$ Overall follow-up at 5 years: $81.8 \%$.

in the ODI score at 2 years $(77.2 \%)$ compared with fusion patients $(64.8 \%$; $\mathrm{p}<0.05)$. Similarly, at 2 years, a significantly greater percentage of TDR patients had a $\geq$ 15 -point improvement in the ODI score (67.8\% of TDR patients and $54.9 \%$ of fusion patients, $\mathrm{p}=0.0449)$.

At 5 years, both treatment groups maintained significant improvements in the ODI score compared with baseline $(p<0.0001)$. The mean ODI score improvements for TDR patients were maintained from 2 to 5 years, whereas mean ODI improvements for fusion patients were similar to those for TDR patients at 5 years $(p=0.4552)$. The majority of patients in both treatment groups had ODI score improvements $\geq 15 \%$ at 5 years $(76.5 \%$ of fusion patients, $78.6 \%$ of TDR patients, $\mathrm{p}=0.8417$ ) or $\geq 15$ points at 5 years $(62.8 \%$ of fusion patients, $74.6 \%$ of TDR patients, $\mathrm{p}=0.1427)$. Of the patients who had ODI score improvements $\geq 15 \%$ at 2 years compared with baseline, a substantial proportion of patients maintained $\geq 15 \%$ improvement from baseline at 5 years $(86.1 \%$ of fusion patients and $89.1 \%$ of TDR patients).

36-Item Short Form Health Survey PCS. The mean baseline SF-36 PCS was similar in TDR and fusion patients (Table 5). Both treatment groups had improvements in the SF-36 PCS at 2 and 5 years of follow-up, compared with baseline $(\mathrm{p}<0.0001)$. The TDR patients experienced significantly greater improvements in SF-36 PCSs at 2 years than did fusion patients $(\mathrm{p}=0.0363)$; a similar trend was observed at 5 years $(p=0.1677)$. At 2 years, $70.0 \%$ of fusion patients and $79.2 \%$ of TDR patients had maintained or improved SF-36 PCSs ( $p=0.0943)$. At 5 years, the majority of patients maintained or improved SF-36 PCSs compared with baseline $(74.0 \%$ of fusion patients and $81.3 \%$ of TDR patients, $\mathrm{p}=0.3054)$. Of the patients who maintained or improved SF-36 PCSs at 2 years compared with baseline, a substantial proportion of them also maintained or improved scores at 5 years compared with baseline $(81.1 \%$ of fusion patients and $88.2 \%$ of TDR patients).

Neurological Success. Neurological success was defined as the maintenance or improvement of patient responses to all neurological criteria: sensory and motor status, reflexes, and straight-leg test. At 2 years posttreatment, the TDR group was statistically superior to the fusion group, with $91.2 \%$ success (135 of 148 patients) compared with $81.4 \%$ (57 of 70 patients), respectively ( $p=0.0341$ ). Compared with rates at 2 years, the percentage of patients achieving overall neurological success at 5 years was similar in the TDR patients (111 [88.8\%] of 125 patients) and increased in fusion patients (43 [89.6\%] of 48 patients); there was no significant difference in neurological success between TDR and fusion patients at 5 years posttreatment $(\mathrm{p}=1.0000)$. Of the patients who had neurological success at 2 years, $90.5 \%$ of fusion patients and $93.0 \%$ of TDR patients had neurological success at 5 years.

Radiographic Success. The composite statistical success definition includes 6 radiographic outcomes. Five of these components refer to qualitative evaluations of device migration, device subsidence, disc height maintenance, fusion status, and radiolucency (Table 6). The results presented below refer to patients with available radiographs for the 5-year follow-up visit. Although clinical follow-up at 5 years was $81.8 \%$ of 236 patients, only $72.9 \%$ of the patients had complete radiographic data sets and are included in this subanalysis. In the remaining patients, radiographs were deemed not satisfactory for digital analysis.

Three TDR patients demonstrated radiographic evi- 
Five-year results of an FDA investigational device exemption study

TABLE 3: Summary of demographic and baseline characteristics in 236 patients enrolled and treated ${ }^{*}$

\begin{tabular}{lccc}
\hline \multirow{2}{*}{ Parameter } & \multicolumn{2}{c}{ No. (\%) } & \\
\cline { 2 - 3 } & Fusion Group & TDR Group & p Value $\dagger$ \\
\hline no. of patients & 75 & 161 & \\
mean age at surgery in yrs & $40.4 \pm 7.6$ & $38.7 \pm 8.0$ & 0.1299 \\
no. of males & $34(45.3)$ & $82(50.9)$ & 0.4849 \\
race & & & 0.6239 \\
$\quad$ white & $59(78.7)$ & $133(82.6)$ & \\
African American & $5(6.7)$ & $5(3.1)$ & \\
Hispanic & $10(13.3)$ & $18(11.2)$ & \\
Asian American & $0(0.0)$ & $2(1.2)$ & \\
$\quad$ other & $1(1.3)$ & $3(1.9)$ & \\
smoking status & & & 0.1995 \\
never & $34(45.3)$ & $87(54.0)$ & \\
former & $17(22.7)$ & $40(24.8)$ & \\
current & $24(32.0)$ & $34(21.1)$ & \\
mean BMl in kg/m² & $27.3 \pm 4.4$ & $26.7 \pm 4.3$ & 0.4844 \\
mean ODI score (\%) & $62.7 \pm 10.3$ & $63.4 \pm 12.6$ & 0.6125 \\
narcotics as prior treatment & $57(76.0)$ & $135(83.9)$ & 0.1554 \\
engaged in recreational ac- & $37(49.3)$ & $68(42.2)$ & 0.3272 \\
tivity & & & \\
implant level & & & 0.6612 \\
L3-4 & $3(4.0)$ & $3(1.9)$ & \\
L4-5 & $22(29.3)$ & $54(33.5)$ & \\
L5-S1 & $50(66.7)$ & $104(64.6)$ & \\
prior surgical treatment & $23(30.7)$ & $57(35.4)$ & 0.5552 \\
\hline
\end{tabular}

* A modified version of this table was published in Semin Spine Surg, Vol 24, Zigler JE, Five-year results of the ProDisc-L multicenter, prospective, randomized, controlled trial comparing ProDisc-L with circumferential spinal fusion for single-level disabling degenerative disk disease, 25-31, Copyright Elsevier, 2012. Abbreviation: BMI = body mass index.

$\dagger$ Continuous and ordinal variables were analyzed using a Wilcoxon rank-sum test, and categorical variables were analyzed using the Fisher exact test to compare F1R (fusion 1-level randomized) with P1R (ProDisc 1-level randomized). dence of device migration at 5 years; in all 3 of these patients, radiographic evidence of migration was first noted at the 3-month follow-up visit. There were 2 cases of TDR radiographic subsidence by the 5-year follow-up. In one of these patients, the radiographic subsidence was present at the 3-month visit. In the other patient, there was evidence of radiolucency prior to the 2-year visit; radiographs at the 5-year visit showed evidence of TDR subsidence with a concomitant decrease in disc height. There were no additional cases of radiolucency, subsidence, or disc height changes in TDR patients with available radiographs at 5 years.

At 5 years, none of the fusion patients with available films demonstrated device migration. Four fusion patients had a disc height decrease more than $3 \mathrm{~mm}$ (due to subsidence or graft settling) at the 5-year visit. No cases of subsidence (that is, violation of vertebral endplates $>3$ $\mathrm{mm}$ ) or radiolucency were observed in any of the fusion patients with available radiographs at 5 years.

Radiographic films available at 5 years indicated that 2 fusion patients had failed to attain more than $50 \%$ trabecular bridging bone, although none of the fusion patients required a secondary procedure for clinical pseudarthrosis. Radiographic fusion rates were $97.1 \%$ and $95.8 \%$ of fusion patients with available films at 2 and 5 years, respectively. All fusion patients had less than $3 \mathrm{~mm}$ of translation and less than $5^{\circ}$ of flexion and extension rotation on 5-year radiographs.

The sixth radiographic outcome was flexion and extension ROM, defined in the TDR group as restoration to a normal ROM at the implanted level (for L3-4 and L4-5, between $6^{\circ}$ and $20^{\circ}$; for L5-S1, between $5^{\circ}$ and $20^{\circ}$ ). The ROM criterion was met by $93.7 \%$ of the TDR patients at 2 years posttreatment and $91.9 \%$ of them at 5 years. The mean flexion and extension ROM in the TDR group was $7.7^{\circ} \pm 4.7^{\circ}$ at 2 years and $7.2^{\circ} \pm 4.1^{\circ}$ at 5 years.

Index-Level Secondary Surgeries. Secondary surgeries at the index level occurred in 9 fusion patients $(12 \%)$ and 13 TDR patients (8\%) by the end of the 5-year study. Details regarding these surgeries, as well as information related to their classification with respect to the primary end point (that is, device success), are provided below.

Device success was defined as the absence of any reoperation to modify or remove implants and no need for supplemental fixation. At 2 years, device success was

TABLE 4: Baseline, 24-month, and 60-month ODI scores*

\begin{tabular}{|c|c|c|c|c|c|c|c|}
\hline \multirow[b]{2}{*}{ Time } & \multicolumn{3}{|c|}{ Fusion Group } & \multicolumn{3}{|c|}{ TDR Group } & \multirow[b]{2}{*}{$\mathrm{p}$ Value } \\
\hline & $\begin{array}{l}\text { No. of } \\
\text { Patients }\end{array}$ & Mean Score (\%) & $\begin{array}{l}\text { Mean \% Change } \\
\text { in Score }\end{array}$ & $\begin{array}{c}\text { No. of } \\
\text { Patients }\end{array}$ & Mean Score (\%) & $\begin{array}{l}\text { Mean \% Change } \\
\text { in Score }\end{array}$ & \\
\hline baseline & 75 & $62.7 \pm 10.3$ & - & 161 & $63.4 \pm 12.6$ & - & 0.6125 \\
\hline 2 yrs & 71 & $39.8 \pm 24.3$ & $-37.8 \pm 36.0$ & $149 \ddagger$ & $34.5 \pm 24.5$ & $-47.4 \pm 34.8$ & 0.0550 \\
\hline $5 \mathrm{yrs}$ & 51 & $36.2 \pm 25.7$ & $-43.8 \pm 37.1$ & 126 & $34.2 \pm 24.3$ & $-47.5 \pm 34.7$ & 0.4552 \\
\hline
\end{tabular}

* A modified version of this table was published in Semin Spine Surg, Vol 24, Zigler JE, Five-year results of the ProDisc-L multicenter, prospective, randomized, controlled trial comparing ProDisc- $L$ with circumferential spinal fusion for single-level disabling degenerative disk disease, 25-31, Copyright Elsevier, 2012.

† Wilcoxon test was used to compare baseline data, and t-tests were used to compare 24- and 60-month percent improvement. $\ddagger$ Patients with device failures were excluded. 
J. E. Zigler and R. B. Delamarter

TABLE 5: 36-Item Short Form Health Survey physical component scores*

\begin{tabular}{lcccccccc}
\hline & \multicolumn{4}{c}{ Fusion Group } & & \multicolumn{3}{c}{ TDR Group } \\
\cline { 2 - 3 } Time & $\begin{array}{c}\text { No. of } \\
\text { Patients }\end{array}$ & Mean Score & $\begin{array}{c}\text { Mean \% Change } \\
\text { in Score }\end{array}$ & & $\begin{array}{c}\text { No. of } \\
\text { Patients }\end{array}$ & Mean Score & $\begin{array}{c}\text { Mean \% Change } \\
\text { in Score }\end{array}$ & p Value† \\
\hline baseline & 74 & $30.9 \pm 5.6$ & - & & 158 & $31.1 \pm 6.5$ & - & 0.7394 \\
2 yrs & $70 \ddagger$ & $38.8 \pm 11.3$ & $29.8 \pm 40.9$ & & $147 \ddagger$ & $42.8 \pm 11.1$ & $39.4 \pm 43.5$ & 0.0363 \\
5 yrs & 51 & $40.1 \pm 13.6$ & $29.9 \pm 43.7$ & & 126 & $42.0 \pm 11.3$ & $40.1 \pm 43.9$ & 0.1677 \\
\hline
\end{tabular}

* A modified version of this table was published in Semin Spine Surg, Vol 24, Zigler JE, Five-year results of the ProDisc-L multicenter, prospective, randomized, controlled trial comparing ProDisc-L with circumferential spinal fusion for single-level disabling degenerative disk disease, 25-31, Copyright Elsevier, 2012.

$\dagger$ Wilcoxon test was used to compare baseline data, t-tests used to compare 2-year and 5-year percent improvement.

‡ Some patients had incomplete data sets.

achieved in $96.3 \%$ of the 161 TDR patients and $97.3 \%$ of the 75 fusion patients $(\mathrm{p}=1.0000)$. Six TDR patients (3.7\%) were considered to have device failures at 2 years: 2 cases of polyethylene migration due to extreme trauma (discussed below), 1 polyethylene inlay migration within 48 hours that was not locked correctly at the time of implantation, and 1 case of implant migration as a result of oversizing. The implant migration event led us to add an exclusion criterion requiring a minimum vertebral body dimension less than $34.5 \mathrm{~mm}$ in the mediolateral direction and/or less than $27 \mathrm{~mm}$ in the anteroposterior direction (the exact size of the smallest available ProDisc-L endplate), as measured on a preoperative axial CT scan obtained in small-stature patients. This size exclusion was not required for any other potential patient in the randomized study. The 2 remaining TDR device failures that occurred by the 2-year follow-up included 1 case of technical error in which the inlay was inserted backward, requiring reoperation and reinsertion of the inlay, and 1 case in which a patient required supplemental posterior fixation due to unresolved pain. One additional TDR patient had secondary surgery at the index level but was not

TABLE 6: Radiographic outcomes at the 5-year follow-up*

\begin{tabular}{llll}
\hline & \multicolumn{2}{c}{ No./Total (\%) } & \\
\cline { 2 - 3 } Radiographic Component & Fusion Group & TDR Group & p Value† \\
\hline no device migration & $48 / 48(100.0)$ & $121 / 124(97.6)$ & 0.5607 \\
no device subsidence & $48 / 48(100.0)$ & $122 / 124(98.4)$ & 1.0000 \\
disc height decrease $\leq 3$ & $44 / 48(91.7)$ & $121 / 123(98.4) \ddagger$ & 0.0530 \\
$\quad$ mm & & & \\
fusion status & $46 / 48(95.8)$ & $124 / 124(100.0)$ & 0.0767 \\
no radiolucency & $48 / 48(100.0)$ & $124 / 124(100.0)$ & 1.0000 \\
ROM & $47 / 47(100.0) \S$ & $113 / 123(91.9) \ddagger$ & 0.0634 \\
\hline
\end{tabular}

* A modified version of this table was published in Semin Spine Surg, Vol 24, Zigler JE, Five-year results of the ProDisc-L multicenter, prospective, randomized, controlled trial comparing ProDisc-L with circumferential spinal fusion for single-level disabling degenerative disk disease, 25-31, Copyright Elsevier, 2012.

$\dagger$ Comparing TDR and fusion using the Fisher exact test.

$\ddagger$ One TDR patient was missing baseline disc height and range of motion measurements due to missing or poor-quality radiographs.

$\S$ One fusion patient was missing extension radiographs. considered to have device failure. This patient underwent hemilaminotomy and discectomy with nerve root decompression that did not require modification or explantation of the device.

One case of traumatic polyethylene component extrusion occurred at L5-S1 in a 33-year-old male powerlifter who worked out regularly, lifting 700-lb weights, and was discovered at the 18 -month postoperative visit. It had occurred several weeks earlier when the previously asymptomatic patient experienced acute pain while working out. He sustained an iliac vein laceration during revision surgery and a postoperative compartment syndrome with complications. His device implantation was revised to a fusion.

The second case of traumatic polyextrusion occurred at L5-S1 in a 41-year-old man who was involved in a motor vehicle accident 23 months after implantation. While experiencing increased back pain after the accident, this patient fell in the shower, causing a further increase in pain. As a result of these sequential traumas, the polyethylene component was noted to have extruded anteriorly. Device implantation was revised to a $360^{\circ}$ fusion, and the patient did not show significant clinical improvement from his preoperative status.

At 2 years posttreatment, 2 fusion patients $(2.7 \%)$ were considered to have device failures when both patients had unresolved pain requiring reoperation. By 2 years posttreatment, another 2 fusion patients underwent hardware removal for hardware-related pain and were not considered to have device failures.

At 5 years posttreatment, device success was achieved in $93.2 \%$ of 161 TDR patients and $93.3 \%$ of 75 fusion patients (cumulative). The 5 TDR patients who were classified as having device failures between 3 and 5 years of follow-up underwent supplemental fixations at the index level due to pain, and the fixations were addressed posteriorly. An additional TDR patient underwent secondary surgery at the index level between 3 and 5 years of follow-up and was not considered to have device failure; this patient underwent hemilaminectomy nerve decompression along with the removal of a small disc fragment at the index level.

Between 3 and 5 years of follow-up, 3 fusion patients were considered to have device failures because they had unresolved pain that required reoperation (6.7\% cumulative). Two additional fusion patients had index-level sur- 


\section{Five-year results of an FDA investigational device exemption study}

geries but were not considered to have device failures because the device or intended treatment was not altered in any way. These 2 fusion patients underwent routine hardware removal for pain; the fusion mass in these patients was confirmed to be solid during the surgery.

\section{Secondary Clinical Outcomes}

VAS Pain. A summary of VAS pain assessment is provided in Table 7. Both TDR and fusion groups demonstrated significant improvements in VAS pain scores at 2 and 5 years posttreatment compared with baseline (p $<0.0001)$. The mean percentage improvements in VAS pain were similar in TDR and fusion patients at the 2- and 5-year follow-up visits.

VAS Satisfaction. Patients indicated their satisfaction with treatment on a $100-\mathrm{mm}$ VAS. At 2 years posttreatment, mean VAS satisfaction was statistically greater in TDR patients than in fusion patients $(76.7 \pm 29.2$ vs 67.3 \pm 31.5 , respectively, $\mathrm{p}=0.0150$ ). At 5 years posttreatment, TDR patient satisfaction was similar to that at 2 years, whereas mean VAS satisfaction in fusion patients increased to a level that was similar to that in TDR patients (TDR: $78.3 \pm 27.1$, fusion: $78.1 \pm 26.7, p=0.6199)$. When asked if they would have the same surgery again, $82.5 \%$ of TDR patients and $68.0 \%$ of fusion patients responded "yes" at 5 years posttreatment; this rate was not significantly different between the groups $(\mathrm{p}=0.1634)$.

Recreational Activity Status. At baseline, a similar proportion of TDR and fusion patients reported that they engaged in recreational activity (42.2\% of 161 TDR patients and $49.3 \%$ of 75 fusion patients). At 2 years, the recreational status improved for both groups and trended toward a greater percentage of TDR patients engaging in recreational activity compared with fusion patients $(88.4 \%$ of 147 TDR patients vs $78.3 \%$ of 69 fusion patients, $\mathrm{p}=$ $0.0640)$. At 5 years, the recreational activity status for both treatment groups had improved compared with baseline and was not significantly different (82.4\% of 125 TDR patients vs $90.0 \%$ of 50 fusion patients, $\mathrm{p}=0.2526$ ).

Narcotic Use. At the time of surgery, $76 \%$ of fusion patients and $84 \%$ of TDR patients had used narcotics as a form of prior conservative treatment. At 2 years (42.5\% of 70 fusion patients and $44.6 \%$ of 148 TDR patients) and 5 years $(40.0 \%$ of 50 fusion patients and $38.4 \%$ of 125 TDR patients), the percentage of patients taking narcotics had decreased from baseline.

\section{Alternate Analyses}

While reviewing the study data, an alternate analysis was requested by the FDA to incorporate changes to the definition of statistical success that was not part of the original study protocol. This modified definition of statistical success was identical to that described in Table 1, except that the condition for ODI improvement from baseline was 15 points (instead of 15\%) and the condition for ROM success was any increase in ROM from baseline for TDR patients (rather than a return to a normal range). The FDA also requested a more conservative noninferiority margin of $10 \%$. Using this alternate definition, we calculated that $40.8 \%$ of fusions and $53.4 \%$ of TDRs were statistical successes at 2 years and $41.1 \%$ of fusions and $48.1 \%$ of TDRs were statistical successes at 5 years. Statistical noninferiority (10\% margin) was demonstrated at both follow-up periods using this alternate definition $(\mathrm{p}<0.05)$.

We also conducted an intent-to-treat analysis for the 5-year exploratory clinical outcomes (Table 8). In the intent-to-treat analysis, we compared ODI, SF-36, and VAS pain scores at the 5-year follow-up in patients randomized to the TDR or fusion group, regardless of the type of intervention that was actually administered. This analysis included the 6 protocol violators during the index procedure (5 randomized to fusion, 1 randomized to TDR), as well as 11 TDR patients and 5 fusion patients who had device failures by 5 years. We also evaluated patients with missing data at the 5-year follow-up using the last observation carried forward (LOCF) method. All of these patients were included in the intent-to-treat analysis. No differences in the trends were observed in the intent-totreat analyses compared with those observed in the perprotocol analysis.

\section{Adverse Events}

On completion of the study, the number of adverse events reported per patient was similar for the fusion and TDR treatment groups (5.1 and 5.4 per patient, respectively, $\mathrm{p}=0.5072$ ). However, the fusion group had a significantly greater number of reported severe or lifethreatening adverse events compared with the TDR group ( 0.58 and 0.38 per patient, respectively, $\mathrm{p}=0.0364$ ).

Complications during the index surgery included 2 fusion group patients who experienced clinically signifi-

TABLE 7: Patient pain assessment using the 100-mm VAS*

\begin{tabular}{lcccccccc}
\hline & \multicolumn{3}{c}{ Fusion Group } & \multicolumn{5}{c}{ TDR Group } \\
\cline { 2 - 3 } Time & $\begin{array}{c}\text { No. of } \\
\text { Patients }\end{array}$ & Mean Score & Mean \% Change & $\begin{array}{c}\text { No. of } \\
\text { Patients }\end{array}$ & Mean Score & Mean \% Change & p Value† \\
\hline baseline & 73 & $74.9 \pm 14.7$ & - & 159 & $75.9 \pm 16.4$ & - & 0.3595 \\
2 yrs & 71 & $43.3 \pm 31.6$ & $-42.4 \pm 42.9$ & 149 & $36.6 \pm 30.1$ & $-49.9 \pm 41.9$ & 0.1340 \\
5 yrs & 51 & $40.0 \pm 32.1$ & $-47.5 \pm 43.8$ & 125 & $37.1 \pm 29.3$ & $-48.7 \pm 44.6$ & 0.5668 \\
\hline
\end{tabular}

* A modified version of this table was published in Semin Spine Surg, Vol 24, Zigler JE, Five-year results of the ProDisc-L multicenter, prospective, randomized, controlled trial comparing ProDisc- $L$ with circumferential spinal fusion for single-level disabling degenerative disk disease, 25-31, Copyright Elsevier, 2012.

† Wilcoxon test was used to compare baseline data, and t-tests were used to compare 24- and 60-month percent improvement. 
J. E. Zigler and R. B. Delamarter

TABLE 8: Five-year intent-to-treat analysis, including major protocol violators and device failures*

\begin{tabular}{|c|c|c|c|c|c|c|c|}
\hline \multirow[b]{2}{*}{ Outcome } & \multicolumn{3}{|c|}{ Fusion } & \multicolumn{3}{|c|}{ TDR } & \\
\hline & \multicolumn{3}{|c|}{ Success/Total (\%) } & \multicolumn{3}{|c|}{ Success/Total (\%) } & p Value \\
\hline \multicolumn{8}{|l|}{ primary } \\
\hline statistical success & \multicolumn{3}{|c|}{$26 / 55(47.3)$} & \multicolumn{3}{|c|}{$72 / 135(53.3)$} & 0.5226 \\
\hline statistical success, LOCF & \multicolumn{3}{|c|}{$34 / 80(42.5)$} & \multicolumn{3}{|c|}{$84 / 162(51.9)$} & 0.1754 \\
\hline Outcome & $\begin{array}{c}\text { No. of } \\
\text { Patients }\end{array}$ & Mean Score & Mean \% Change & $\begin{array}{c}\text { No. of } \\
\text { Patients }\end{array}$ & Mean Score & Mean \% Change & p Value§ \\
\hline \multicolumn{8}{|l|}{ secondary } \\
\hline ODI (\%) & 60 & $38.5 \pm 25.8$ & $-41.3 \pm 37.3$ & 134 & $34.6 \pm 24.5$ & $-46.7 \pm 35.3$ & 0.1715 \\
\hline ODI, LOCF (\%)‡ & 80 & $40.6 \pm 25.7$ & $-37.2 \pm 38.1$ & 162 & $35.9 \pm 24.7$ & $-44.4 \pm 36.5$ & 0.0809 \\
\hline SF-36 PCS & 58 & $39.4 \pm 13.5$ & $28.4 \pm 42.6$ & 133 & $41.7 \pm 11.4$ & $38.8 \pm 43.7$ & 0.0649 \\
\hline SF-36 PCS, LOCF $\ddagger$ & 80 & $38.7 \pm 13.1$ & $27.5 \pm 42.5$ & 162 & $41.0 \pm 11.5$ & $36.3 \pm 43.8$ & 0.0689 \\
\hline VAS Pain & 60 & $42.3 \pm 31.7$ & $-44.7 \pm 42.8$ & 133 & $37.6 \pm 29.4$ & $-49.0 \pm 41.9$ & 0.2617 \\
\hline VAS Pain, LOCF & 80 & $45.0 \pm 31.6$ & $-41.0 \pm 42.3$ & 162 & $39.6 \pm 30.2$ & $-46.2 \pm 43.0$ & 0.1890 \\
\hline
\end{tabular}

cant blood loss $(>1500 \mathrm{ml})$ and 2 additional patients in the fusion group who experienced dural tears. None of these complications resulted in clinical sequelae. Postoperatively, retrograde ejaculation was reported in 1 fusion and 2 TDR patients. No infections were reported in the TDR group, and 2 posterior wound infections were reported in the fusion group. Deep venous thrombosis developed after surgery in 3 patients ( 2 TDR and 1 fusion) and was successfully treated medically. One TDR patient $(0.6 \%)$ experienced clinically significant blood loss (> 1500 $\mathrm{ml}$ ) during revision surgery. At 5 years posttreatment, 5 deaths had occurred (1 fusion and 4 TDR patients), all for reasons unrelated to the surgery or implants.

\section{Discussion}

This paper builds on results of a 2-year FDA IDE study for ProDisc-L and continues the follow-up of investigational (TDR) and control (fusion) cohorts through a 5-year postapproval study. The primary hypothesis of this multicenter, prospective randomized trial was supported at both 2 and 5 years posttreatment, allowing us to conclude that TDR is noninferior to fusion. Although the study was not designed as a superiority study, a significant difference favoring TDR was found in statistical success rates at 2 years. In addition to the primary outcome, exploratory analyses led to several conclusions. Both TDR and fusion patients in whom an average of 9 months of prior conservative care had failed showed improved clinical outcomes at the 2- and 5-year followups as compared with baseline. At 2 years, TDR patients showed statistically superior results in mean clinical outcomes such as ODI improvement, neurological success, and radiographic maintenance of disc height. The mean clinical outcomes in TDR patients held steady from 2 to 5 years and maintained that improvement compared with baseline. Fusion patients experienced further improvement in mean clinical outcomes from 2 to 5 years, with mean outcomes reaching levels similar to those observed for the TDR patients at 5 years.

An alternate analysis suggested by the FDA, after the study was underway, required a 15 -point ODI improvement rather than $15 \%$ improvement from baseline and changed the definition of ROM success. Although the hard data remain exactly the same, this change in the semantics of success highlights the difficulty in interpreting complex success criteria. Most clinicians would be much more comfortable with an ROM success definition requiring a return to a normal range (the original definition) rather than an increase in the range (for example, a meaningless increase from 0 to $1^{\circ}$ ), as imposed by the FDA; however, both are presented.

In the current study, a successful fusion status required strong evidence of fusion, that is, more than $50 \%$ trabecular bridging bone or bone mass maturation, increased or maintained bone density at the site, and no visible gaps in the fusion mass. Using these strict radiographic criteria, we calculated a $97.1 \%$ fusion rate at 2 years and $95.8 \%$ at 5 years in patients with DDD in whom conservative treatment had failed. These fusion rates compare favorably with those previously reported for instrumented fusion in patients undergoing treatment for DDD in prospective randomized studies $(73 \%-95 \%) .^{3-5,10}$ It is noteworthy that patients in the current study received femoral ring allograft and autogenous iliac crest bone graft but no bone morphogenetic protein and that fusion rates in this series of patients were higher than those reported in prospective randomized studies evaluating bone morphogenetic protein in cases of single-level DDD (for example, $88 \%$ reported by Dimar et al. ${ }^{4}$ and $91 \%$ reported by Burkus et $\mathrm{al}^{2}{ }^{2}$ ). These findings further support the quality of surgical treatment provided to the control group. 


\section{Five-year results of an FDA investigational device exemption study}

Contrary to increasingly strong evidence in the scientific literature supporting the effectiveness of both arthroplasty and fusion in appropriately selected patients, recent surgical approval policies by insurance payers have become narrower, limiting coverage for reconstructive spine surgery in patients who are experiencing persistent functional disability as a result of mechanical DDD. Our results combined with other strong scientific evidence in the peer-reviewed literature indicate a need to reconsider this conservative posture. In the current study, highly disabled patients with mechanical DDD, in whom conservative treatment had failed for at least 6 months, were treated with spinal fusion or TDR. This population was carefully selected using strict inclusion and exclusion criteria. Not only did patients in both treatment groups experience significant improvements in measurable clinical outcomes, such as the ODI score and VAS pain, but there were also substantial improvements in functional status. Specifically, over $80 \%$ of patients in this study experienced improvements in recreational status that were maintained 5 years after the index surgery, indicating substantial improvements in their quality of life that were not afforded by months of conservative care. The percentage of patients utilizing narcotics at the 5-year follow-up visit was $39 \%$, less than half the percentage of patients who had used narcotics as part of failed conservative treatment.

Data from the current study clearly show that in an appropriately selected patient, spinal surgery is a very good treatment option for improving function and ameliorating disability and pain through at least 5 years of follow-up and is beneficial to the patient according to multiple parameters for life quality. Our study hypothesis was supported in that TDR surgery using the ProDisc-L proved to be noninferior to fusion at 2 years posttreatment, and success was maintained through a minimum of 5 years after surgery.

\section{Conclusions}

Measures of disability, pain, and neurological status all improved more quickly in TDR patients than in the fusion group and were similarly improved from baseline in both groups at 5 years posttreatment. Furthermore, narcotics use decreased and recreational status increased in both treatment groups as compared with baseline. The 5 -year results of this study support either TDR or fusion surgery as a predictable and lasting treatment option to improve pain and function in properly selected patients with mechanical DDD. These improvements occurred dramatically immediately after surgery and were maintained through the follow-up period.

\section{Disclosure}

Dr. Delamarter receives royalties from Synthes Spine, Inc. Drs. Zigler and Delamarter are consultants for Synthes Spine, Inc. They also serve on the ProDisc-L Publications Committee but have received no direct financial compensation for the writing and editing of this paper.

Author contributions to the study and manuscript preparation include the following. Conception and design: both authors.
Acquisition of data: both authors. Analysis and interpretation of data: both authors. Drafting the article: both authors. Critically revising the article: both authors. Reviewed submitted version of manuscript: both authors. Approved the final version of the manuscript on behalf of both authors: Zigler.

\section{References}

1. Blackwelder WC: "Proving the null hypothesis" in clinical trials. Control Clin Trials 3:345-353, 1982

2. Burkus JK, Gornet MF, Schuler TC, Kleeman TJ, Zdeblick TA: Six-year outcomes of anterior lumbar interbody arthrodesis with use of interbody fusion cages and recombinant human bone morphogenetic protein-2. J Bone Joint Surg Am 91:1181-1189, 2009

3. Chung SK, Lee SH, Lim SR, Kim DY, Jang JS, Nam KS, et al: Comparative study of laparoscopic L5-S1 fusion versus open mini-ALIF, with a minimum 2-year follow-up. Eur Spine J 12:613-617, 2003

4. Dimar JR, Glassman SD, Burkus KJ, Carreon LY: Clinical outcomes and fusion success at 2 years of single-level instrumented posterolateral fusions with recombinant human bone morphogenetic protein-2/compression resistant matrix versus iliac crest bone graft. Spine 31:2534-2540, 2006

5. Fischgrund JS, Mackay M, Herkowitz HN, Brower R, Montgomery DM, Kurz LT: 1997 Volvo Award winner in clinical studies. Degenerative lumbar spondylolisthesis with spinal stenosis: a prospective, randomized study comparing decompressive laminectomy and arthrodesis with and without spinal instrumentation. Spine (Phila Pa 1976) 22:2807-2812, 1997

6. Fritzell P, Hägg O, Wessberg P, Nordwall A: 2001 Volvo Award Winner in Clinical Studies: Lumbar fusion versus nonsurgical treatment for chronic low back pain: a multicenter randomized controlled trial from the Swedish Lumbar Spine Study Group. Spine (Phila Pa 1976) 26:2521-2534, 2001

7. Hudson-Cook N, Tomes-Nicholson K, Breen A: A revised Oswestry disability questionnaire, in Roland M, Jenner JR (eds): Back Pain: New Approaches to Rehabilitation and Education. Manchester: Manchester University Press, 1989, pp 187204

8. Molinari RW, Gerlinger T: Functional outcomes of instrumented posterior lumbar interbody fusion in active-duty US servicemen: a comparison with nonoperative management. Spine J 1:215-224, 2001

9. Weinstein JN, Lurie JD, Tosteson TD, Tosteson AN, Blood EA, Abdu WA, et al: Surgical versus nonoperative treatment for lumbar disc herniation: four-year results for the Spine Patient Outcomes Research Trial (SPORT). Spine (Phila Pa 1976) 33:2789-2800, 2008

10. Zdeblick TA: A prospective, randomized study of lumbar fusion. Preliminary results. Spine (Phila Pa 1976) 18:983-991, 1993

11. Zigler J, Delamarter R, Spivak JM, Linovitz RJ, Danielson GO III, Haider TT, et al: Results of the prospective, randomized, multicenter Food and Drug Administration investigational device exemption study of the ProDisc-L total disc replacement versus circumferential fusion for the treatment of 1-level degenerative disc disease. Spine (Phila Pa 1976) 32:1155-1163, 2007

Manuscript submitted June 1, 2011.

Accepted September 12, 2012.

Please include this information when citing this paper: published online October 19, 2012; DOI: 10.31712012.9.SPINE11498.

Address correspondence to: Jack E. Zigler, M.D., Texas Back Institute, 6020 West Parker Road, Suite 200, Plano, Texas 75093. email: jzigler@texasback.com. 